\title{
SUR L'ORIGINE DES ALPHAS DE SCISSION (*)
}

\author{
N. CÂRJAN (**) \\ Institut für Kernphysik der Technischen Hochschule Darmstadt, R.F.A.
}

(Reçu le 27 novembre 1975, révisé le 23 février 1976, accepté le 17 juin 1976)

\begin{abstract}
Résumé. - On présente un mécanisme d'émission pour les particules $\alpha$ de scission à partir duquel le spectre énergétique, la fréquence d'apparition et leur variation en fonction de divers paramètres reçoivent une explication unitaire. On discute, en outre, de nouvelles possibilités d'études de la structure et de la dynamique du système fissionnant, au-delà du dernier point-seuil.
\end{abstract}

\begin{abstract}
An emission mechanism for the scission $\alpha$-particles is presented. On this basis, the energy spectrum, the rate of appearance and their variation with different parameters are explained. New possibilities of studying the structure and dynamics of the fissioning system beyond the last saddle-point are also discussed.
\end{abstract}

1. Introduction. - Les investigations sur la fission nucléaire binaire ont élucidé de nombreux aspects relatifs à la transition du noyau de l'état fondamental au dernier point-seuil, mais par contre ont peu expliqué sa descente vers le point de scission. De ce fait, il est évident que tout phénomène connexe qui peut fournir des renseignements sur cette dernière étape est considéré avec grand intérêt.

La fission accompagnée de particules légères représente, apparemment, un admirable exemple d'un tel phénomène car, comme la direction principale d'émission (perpendiculaire à l'axe de fission) de ces noyaux légers le prouve, c'est la scission même qui définit tant le lieu que le moment de leur apparition. En outre, la similitude des caractéristiques des fragments lourds dans le cas de l'émission de ces particules et dans le cas inverse montre qu'il n'est pas question de deux canaux différents et donc que des informations obtenues à partir d'un des processus peuvent être utilisées dans l'autre.

Comment procède-t-on pour extraire les données, que ces particules légères portent, concernant la manière suivant laquelle le noyau se brise ? D'habitude, on paramétrise la configuration des trois fragments au moment de l'émission de la particule légère et on fait varier les paramètres jusqu'à ce que les trajectoires qui y correspondent conduisent à la reproduction des résultats expérimentaux : distributions angulaires et énergétiques.

$\left(^{*}\right)$ Partiellement, ce papier a fait l'objet d'un exposé donné aux Journées d'Etude sur la Fission, Cadarache, France, 21-23 octobre 1974.

(**) Adresse permanente : Institut de Physique Atomique, Bucarest, Roumanie.
Les grandeurs qui définissent la configuration initiale sont représentées dans la figure 1. Dans le cas le plus simple, chacune de ces grandeurs introduit deux paramètres pour caractériser sa distribution : une valeur moyenne et une largeur. Le nombre des paramètres est donc trop grand pour que leur détermination soit suffisamment précise et, comme on l'a remarqué plusieurs fois, ils sont fortement corrélés entre eux, en sorte que tout ajustement sera non univoque. En outre, il n'existe, a priori, aucune raison de supposer une forme donnée pour les distributions.

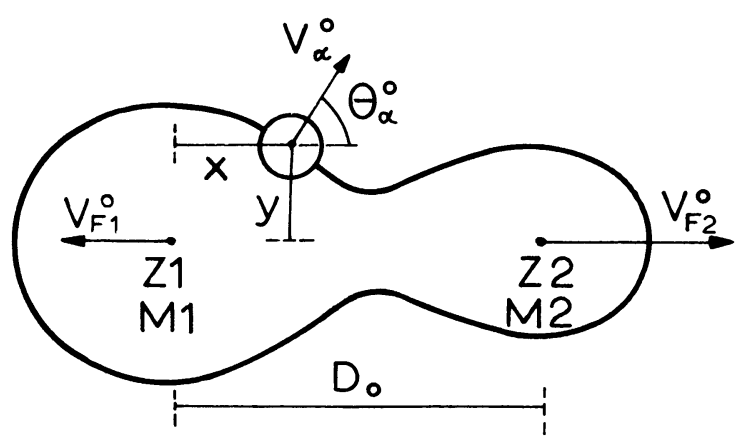

Fig. 1. - Paramètres qui définissent le système fissionnant à l'instant de l'émission de la particule légère.

Pour réduire l'arbitraire dans ce procédé, on essaye dans cet article de comprendre le mécanisme de l'émission des alphas de scission et d'apporter ainsi des informations théoriques supplémentaires concernant la configuration initiale.

Dans l'élaboration d'un tel mécanisme la première question c'est d'établir la position du moment d'émission par rapport au moment de séparation des fragments. 
2. L'hypothèse d'émission avant le moment de scission. - Nous voudrions mentionner quelques considérations qui semblent exclure la possibilité que les particules $\alpha$ de long parcours soient émises après la scission.

2.1 Si le déficit de quatre unités est corrigé, la distribution des masses dans la fission $\alpha$, excepté le fait qu'elle est plus étroite, coïncide dans tous ses aspects avec celle de la fission ordinaire [1, 2].

Mais la probabilité qu'un des fragments émette une particule $\alpha, P_{1}^{\alpha}+P_{2}^{\alpha}$, varie fortement avec la division de masse (et de charge correspondante), et cette variation n'est pas semblable (elle n'a d'ailleurs aucune raison de l'être) à la distribution des masses. Ce comportement reflète en majeure partie la variation d'énergie de liaison de l'alpha avec la masse et la charge du fragment émetteur et il est soutenu par des calculs dans l'hypothèse d'évaporation [3] et est contenu dans la variation de la dépense d'énergie $E_{\mathrm{c}}$ avec la masse et la charge du fragment émetteur dans le modèle non adiabatique [4].

Par conséquent, la distribution des masses ne pourra pas être gardée par une émission postérieure au moment de la scission.

En outre, comme l'émission de la particule $\alpha$ après la scission modifie seulement les propriétés du fragment émetteur, léger ou lourd, toutes les distributions concernant un des fragments devront avoir un caractère double : l'un correspondant au cas où le fragment est émetteur, l'autre au cas contraire. N'ayant pas de raisons de considérer les distributions des fragments émetteurs sensiblement plus étroites que les autres, les distributions résultantes devront être, dès lors, plus larges. Mais en réalité, tous ces spectres (masse, énergie cinétique et neutrons émis) sont plus étroits.

Une émission antérieure à la scission explique fort naturellement cette caractéristique : elle diminue le taux de diffusion des nucléons dans la région du col et (voir plus loin) se fait par le ralentissement du processus de fission.

2.2 Dans la fission induite par neutrons, la classification des rapports $N_{\alpha} / N_{\mathrm{F}}$ en deux groupes correspondant aux diverses résonances [5], est complètement en accord seulement avec la détermination directe du spin $J$ de la résonance et n'est pas du tout corrélable avec la multiplicité neutronique $\bar{v}$ laquelle correspond à l'énergie d'excitation existante.

De ce fait il résulte que cette classification reflète des différences dans la structure, mais non dans la position relative ou dans d'autres caractéristiques des deux états possibles de transition vers la scission. Le moment de scission produisant une transition de phase dans la structure du système, l'émission doit avoir lieu avant ce moment lorsque les informations relatives à la structure du canal de fission se perdent.

2.3 Une émission antérieure est favorisée du point de vue énergétique. Ceci provient de ce que beaucoup d'amas malgré qu'ils soient fortement liés dans les fragments, sont par ailleurs libres dans le système fissionable. D'autre part l'énergie disponible pour l'émission des particules est considérablement plus petite après la scission qu'entre le point-seuil et le point de scission.

$2.4 \mathrm{Si}$ on considère que la similitude des caractéristiques des fragments lourds implique la similitude des configurations de scission, on peut ajouter encore un argument.

La moyenne de l'énergie d'excitation dans la fission spontanée de ${ }^{252} \mathrm{Cf}$ est seulement de 5,5 MeV supérieure à celle du cas où la même fission est accompagnée d'une particule $\alpha$ [2]. Toutefois, dans le cas où l'émission aurait lieu postérieurement, la presque totalité de l'énergie nécessaire (au moins $20 \mathrm{MeV}$ ) devrait être dépensée sur le compte de l'énergie d'excitation des fragments.

Bien que l'énumération précédente soit incomplète, il est clair que les particules alphas de scission sont émises avant la séparation du système fissionable en ses deux fragments.

Cette localisation suggère un mécanisme simple pour leur émission. De quoi s'agit-il ?

3. Un mécanisme possible. - Tous les noyaux qui fissionnent sont des émetteurs alpha dans leur état fondamental. Cette propriété se conserve tout au long du parcours du noyau vers le point de scission, bien sûr continuellement modifié par les nouvelles formes et bilans énergétiques associés à chaque moment de la fission. Une source possible pour les alphas de scission serait donc la désintégration $\alpha$ à partir de la dernière étape du processus de fission. Les particules qui peuvent être émises suivant le mécanisme en cause ne se résument pas au noyau ${ }^{4} \mathrm{He}$, tous les raisonnements qui suivent étant applicables, dans une même mesure, à n'importe quelle structure libre dans le noyau.

Avant tout il est naturel d'essayer de voir, dans une première estimation où une série d'effets considérés comme secondaires seront négligés, quelles sont les prédictions de cette hypothèse pour les principales observables du phénomène.

4. L'origine de la distribution angulaire. - Dans un article précédent [6] on a estimé la probabilité qu'une particule $\alpha$ existe en un certain point $\mathbf{P}$ de la surface du noyau fissionant. En la calculant en divers points, nous avons représenté, figure 2 de la réf. [6], sa distribution qui constitue la distribution primaire de $x$ et $y$.

L'amplitude d'onde de l'alpha a été définie par analogie avec la matrice $R$ par le recouvrement de la fonction d'onde du noyau initial et celle du canal $\alpha$ :

$$
\begin{aligned}
\Phi_{\alpha}(\mathbf{P})=\left[\left(\begin{array}{l}
N \\
2
\end{array}\right)\right. & \left.\left(\begin{array}{l}
Z \\
2
\end{array}\right)\right]^{1 / 2} C_{\alpha} \times \\
& \times \int \Psi_{\mathrm{i}}\left(\boldsymbol{\eta}_{\mathrm{i}}\right) \Psi_{\mathrm{f}}\left(\boldsymbol{\eta}_{\mathrm{f}}\right) \Psi_{\alpha}\left(\boldsymbol{\eta}_{\alpha}\right) \mathrm{d} \boldsymbol{\eta}_{\mathrm{f}} \mathrm{d} \boldsymbol{\eta}_{\alpha}
\end{aligned}
$$


Le modèle à couches avec appariement a été utilisé pour la description des états nucléaires impliqués.

Les trois courbes correspondent au dernier pointseuil et aux deux étapes intermédiaires entre le dernier point-seuil et le point de scission. Prédisant, quand on approche du moment de la scission, la formation des particules $\alpha$ avec préférence pour la région du col, les résultats contiennent dès lors la caractéristique demandée par la direction principale d'émission des particules $\alpha$ au cours de la fission. Cependant, la probabilité de formation aux extrémités du système fissionant n'étant pas nulle, une petite partie des alphas de scission seront émis aussi dans la direction des fragments et pourraient constituer la source de ce qu'on appelle l'émission polaire. A ce point de la discussion, il faut souligner qu'en raison surtout de l'incertitude des paramètres nucléaires, on ne peut attendre que ces courbes représentent davantage qu'une image qualitative.

On peut aussi remarquer que cette variation de la probabilité de formation en fonction de la région de la surface nucléaire est en accord qualitatif avec son analogue classique : la variation du nombre de collisions par unité de temps, contre le mur de potentiel, d'une particule existant dans le noyau.

5. Le bilan énergétique. - L'énergie dont dispose une particule $\alpha$, dans la mesure où elle existe dans un noyau donné, est décrite par :

$$
Q_{\alpha}^{0}=M(Z, A, \varepsilon)-M(Z-2, A-4, \varepsilon)-M(\alpha)
$$

si le noyau considéré n'a pas dépassé le point-seuil. Pour les noyaux fissionables, cette différence reste toujours positive. Pour ${ }^{252} \mathrm{Cf}$, par exemple, elle diminue de $6 \mathrm{MeV}$ dans l'état fondamental à $3,6 \mathrm{MeV}$ au dernier point-seuil et présente ensuite une tendance à remonter [7].

Après le point-seuil l'alpha reçoit aussi une partie de l'énergie potentielle libérée pendant la descente du noyau vers le point de scission jusqu'au moment où, s'approchant du sommet de la barrière, il s'échappe. En conséquence, la distribution des valeurs $Q$ sera donnée par la probabilité $T(Q)$ qu'une particule $\alpha$ ayant l'énergie $Q$ y pénètre, bien sûr à condition qu'elle ne soit pas émise avant d'atteindre cette énergie, c'est-à-dire :

$$
P(Q)=T(Q)\left[1-\sum_{n=1}^{n_{Q}} T\left(E_{n}\right)\right]
$$

où $n_{Q}$ est le nombre de fois que la particule essaye de pénétrer la barrière avant d'atteindre l'énergie $Q$ et $E_{n}$ les énergies qu'elle possède à chaque essai.

Dans la figure 2 sont représentées les barrières vues par la particule $\alpha$ dans un noyau ${ }^{236} \mathrm{U}$ extrêmement déformé. La distance correspondante entre les centres des fragments est proche de celle prédite par les modèles dynamiques au moment de la scission dans le rapport de masse le plus probable (voir réf. [8]).
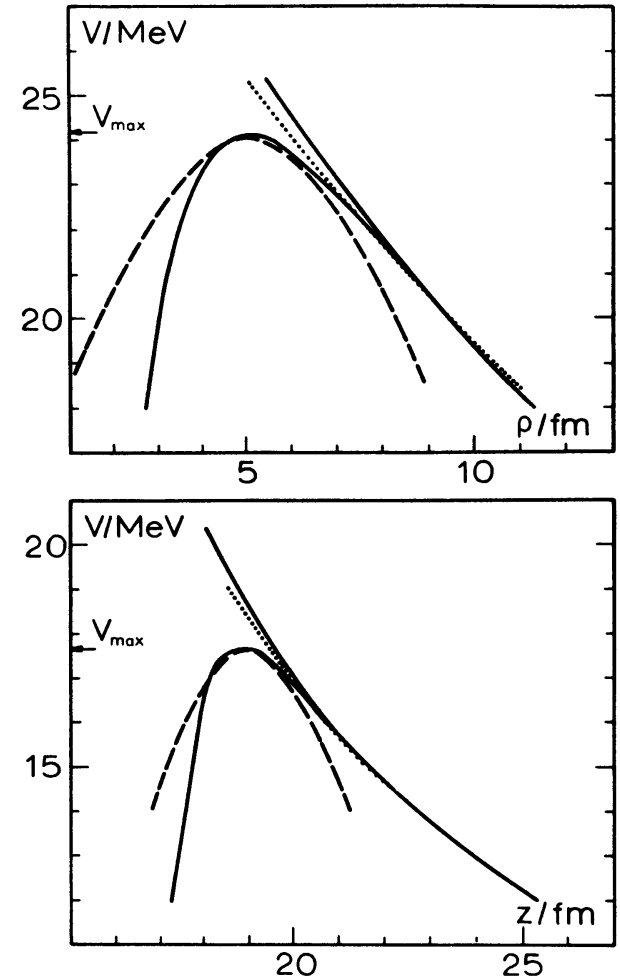

Fig. 2. - Potentiels de la particule $\alpha$ à proximité du moment de la scission au col (en haut) et aux extrémités (en bas). La ligne pointillée représente les potentiels coulombiens correspondant aux fragments ponctuels pour $D_{0}=18,4 \mathrm{fm}$ et la ligne en tirets représente la forme suivant laquelle les barrières ont été approchées lors du calcul de leur transparence.
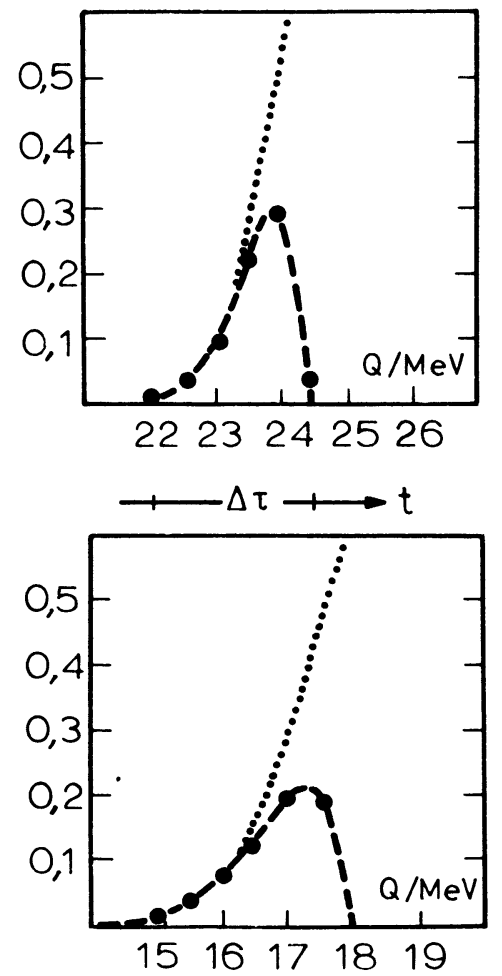

Fig. 3. - Spectres $\alpha$ au moment de l'émission pour une configuration donnée, correspondant aux cas dans lesquels les particules sont émises au col (en haut) et aux extrémités (en bas). La

ligne pointillée représente le coefficient de transmission $T(Q)$. 
Le résultat de l'application de l'équation (2) à ces barrières est montré dans la figure 3 . Un spectre continu s'obtient si on tient compte de la dispersion en énergie de l'onde $\alpha$, en accord avec la relation d'incertitude temps-énergie.

Dans la mesure où l'énergie disponible dépasse la dépense d'énergie $V_{\max }-Q_{\alpha}^{0}\left({ }^{1}\right)$, on prévoit donc une distribution des énergies $\alpha$ dans le système nucléaire légèrement asymétrique autour d'une valeur très proche du sommet de la barrière. Il est à noter que aussi le spectre mesuré présente une telle asymétrie [9].

On peut remarquer la dépendance de la forme du spectre de la diffusivité et de la profondeur du potentiel $\alpha$-nucléaire et on a dès lors une première possibilité d'obtenir des valeurs expérimentales pour ces dernières.

L'équation (2) représente un seul mode de scission et, pour obtenir la forme du spectre mesuré, il faudra donc sommer sur tous les modes possibles :

$$
N(Q)=\sum_{i} \omega_{i} T^{i}(Q)\left[1-\sum_{n=1}^{n^{i} Q} T^{i}\left(E_{n}\right)\right]
$$

où $\omega_{i}$ est le poids du mode $i\left({ }^{2}\right)$.

$\mathrm{La}$ largeur du spectre $\alpha$ réflète donc, comme la largeur du spectre d'énergie cinétique des fragments, la distribution des élongations $D_{0}$ et des énergies cinétiques $E_{\mathrm{F}}^{0}$.

Ignorant le rapport dans lequel ces deux causes interviennent on ne peut actuellement calculer avec précision cette largeur. Supposant qu'il est aussi possible qu'à un tel $E_{\mathrm{F}}$ correspondent plusieurs couples $\left(D_{0}, E_{\mathrm{F}}^{0}\right)$, il serait très utile de mesurer le spectre $\alpha$ pour un tel $E_{\mathrm{F}}$ et un rapport de masses donné $R$ puisque la variation de $\left(D_{0}, E_{\mathrm{F}}^{0}\right)$ ne modifie, ni dans le même sens, ni dans la même mesure, les valeurs de $E_{\mathrm{F}}$ et de $Q_{\alpha}$.

Pour obtenir quand même un ordre de grandeur, les deux causes possibles de la déviation standard $\sigma_{E_{\mathrm{F}}}^{R}$ par rapport à l'énergie cinétique totale la plus probable des fragments pour un $R$ donné ont été analysées indépendamment :

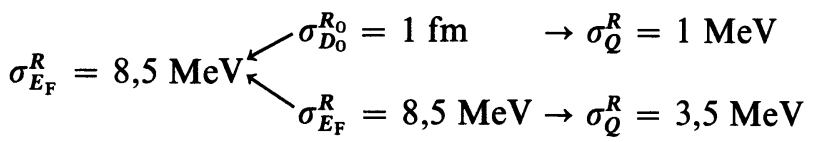

( $\left.{ }^{1}\right)$ En accord avec les calculs du modèle de la goutte liquide pour la différence d'énergie entre le point-seuil et le point de scission [10], les noyaux avec de petits paramètres de fissibilité (c'est-àdire ayant déjà une forme avec un col marqué au point-seuil) vont se séparer avant que la particule $\alpha$ n'atteigne le sommet de la barrière. Dans le cas de l'émission équatoriale, cet effet se fera sentir dès que les noyaux seront plus légers que l'uranium.

$\left({ }^{2}\right)$ La distribution des masses donnant une idée de la distribution de ces poids, il serait possible que les noyaux de transition qui présentent une distribution de masse caractéristique à trois pics, aient un spectre à deux bosses. et considérées comme des limites. A ces valeurs on devra ajouter les largeurs supplémentaires dues à la largeur naturelle des états virtuels $\alpha$, à la largeur du spectre $Q$ (Fig. 3), à la variation de $Q$ en fonction de $R$ et à la distribution du point d'émission.

Revenant à la première figure, on remarque que nous avons maintenant à notre disposition des procédés permettant d'évaluer toutes les distributions liées à la particule $\alpha$.

6. Le spectre mesuré. - A cause du mouvement simultané des fragments lourds, les particules de la région du col ne peuvent pas utiliser, pour leur accélération, tout le potentiel existant au moment de leur émission (Fig. 3). Par conséquent, la distribution de leur énergie cinétique finale $E_{\alpha}$ sera déplacée vers les valeurs plus basses. On s'attend à un effet contraire pour les particules qui bougent dans la même direction que les fragments.

Pour étudier quantitativement cet effet, on a pris la situation de la figure 2 comme configuration initiale et on a calculé les trajectoires des fragments et de la particule $\alpha$ émise au col ou aux extrémités, en résolvant le mouvement des trois charges ponctuelles dans le champ coulombien mutuel. Dans la présente hypothèse, $V_{\alpha}^{0}$, qui est donnée par la vitesse de déplacement de la surface nucléaire (disparition de col ou mouvement des extrémités), dépend de $V_{\mathrm{F}}^{0}$ et a été pris : $V_{\alpha}^{0}(\mathrm{col})=-V_{\mathrm{F}}^{0}$ et $V_{\alpha}^{0}$ (extrémités) $=V_{\mathrm{F}}^{0}$, dans une première approximation. Ce choix a été suggéré par les résultats des calculs dynamiques pour la fission nucléaire $[10,11]$.

Dans ces conditions, une énergie cinétique totale des fragments au moment d'émission de $6 \mathrm{MeV}$ (ou $4 \mathrm{MeV}$ ) explique la valeur $\bar{E}_{\alpha}=16 \mathrm{MeV}$ (ou $23 \mathrm{MeV}$ ) de l'énergie à l'infini des particules détectées perpendiculairement à (ou le long de) l'axe de fission. On voit donc que du point de vue énergétique, on ne peut pas exclure non plus la possibilité que toutes les particules $\alpha$ de long parcours, quel que soit leur point d'apparition, soient émises dans la même étape du processus de fission.

Le mouvement des fragments lourds n'a pas seulement pour effet de déplacer la distribution $Q$ mais aussi de la rétrécir : les particules émises du col (des extrémités) perdent (gagnent) d'autant moins (plus) d'énergie qu'est plus grande la distance à laquelle elles quittent la barrière, c'est-à-dire qu'est plus petite la valeur de $Q$.

7. La fréquence d'apparition. - En liaison directe avec le bilan énergétique, on peut évaluer le taux de production des alphas de scission.

La quantité mesurée dans ce processus, laquelle peut donner des informations concernant sa fréquence, est le rapport $N_{\alpha} / N_{\mathrm{F}}$ du nombre des particules $\alpha$ au nombre des fissions. Ce rapport a été identifié avec $\lambda_{\alpha} / \lambda_{\mathrm{F}}$ en considérant qu'il s'agit d'un processus à branchements. 
Mais dans le cas où la fission et la désintégration $\alpha$ se produisent simultanément, comme c'est le cas dans l'hypothèse présente, l'interprétation de ce rapport est différente. Le nombre $N_{\mathrm{F}}$ des noyaux qui fissionnent, constituant l'ensemble des noyaux dont on suit la désintégration $\alpha, N_{\alpha} / N_{\mathrm{F}}$ représente justement la probabilité qu'un tel noyau émette une particule $\alpha$ de long parcours pendant sa scission. Il est donc donné par la loi fondamentale de la désintégration radioactive :

$N_{\alpha} / N_{\mathrm{F}}=\int_{0}^{\tau} \lambda_{\alpha}(t)\left[1-\sum_{n=1}^{n_{t}} T\left(E_{n}\right)\right] \mathrm{d} t \quad$ pour $\quad \lambda_{\alpha} \tau \ll 1$

où $\tau$ est le temps de scission (c'est en effet le temps de mesure qui est fixé cette fois par la dynamique du processus de fission).

Séparant dans la constante de désintégration $\alpha$, les deux probabilités qui la composent : celle qu'un amas $\alpha$ existe à la surface du noyau et celle que cet amas pénètre la barrière coulombienne nucléaire, l'équation (3) devient, pour un certain mode de scission

$$
N_{\alpha} / N_{\mathrm{F}}=\frac{1}{\hbar} \delta^{2} \int_{0}^{\tau} P[Q(t)] \mathrm{d} t
$$

$N_{\alpha} / N_{\mathrm{F}}$ varie avec la configuration du noyau au moment de l'émission seulement dans la mesure où $\delta^{2}$ varie, donc extrêmement peu.

L'ignorance de la dépendance temporelle du transfert d'énergie vers la particule $\alpha$ peut être évitée en choisissant un intervalle de temps durant lequel la plupart des particules sont émises et en approchant l'intégrale par un triangle de base $\Delta \tau$ :

$$
N_{\alpha} / N_{\mathrm{F}}=\frac{1}{\hbar} \delta^{2} T\left(V_{\max }\right)\left[1-\sum_{n=1}^{n_{V_{\max }}} T\left(E_{n}\right)\right] \frac{\Delta \tau}{2}
$$

Comme on le remarque à partir de la figure 2 de la réf. [6] et comme cela a déjà été discuté dans la réf. [6], pour que la plupart des particules préformées soient détectées perpendiculairement à l'axe de fission, c'est-à-dire pour pouvoir constituer les $\alpha$ de long parcours, ceux-ci doivent quitter les noyaux au cours de la dernière étape du processus de fission, par exemple pendant le passage du système de la déformation $\varepsilon=0,900$ à $\varepsilon=0,985$. Cet intervalle de temps estimé de nouveau à partir des résultats du modèle dynamique de la goutte liquide $[10,11]$ est d'environ de $5 \times 10^{-22} \mathrm{~s}$ et semble, à première vue, exagérément court pour que les particules soient émises en quantité détectable.

Mais l'équation (4) démontre que ce même intervalle de temps explique aussi la valeur expérimentale du rapport $N_{\alpha} / N_{\mathrm{F}}$. En effet, utilisant :

- la valeur expérimentale de la largeur réduite de la désintégration $\alpha$ de l'état fondamental de ${ }^{236} \mathrm{U}$ [12] : $\delta^{2}=1,6 \times 10^{-2} \mathrm{MeV}$, qui est d'ailleurs une valeur typique pour tous les noyaux mesurés jusqu'à présent, - le fait que la valeur intégrale de la probabilité de formation ne varie pas sensiblement avec la déformation [6],

$$
\begin{aligned}
& \text { - les résultats de la figure } 3 \text { et } \\
& -\Delta \tau=5 \times 10^{-22} \mathrm{~s} \text {, il en résulte : } \\
& \qquad N_{\alpha} / N_{\mathrm{F}}=1,8 \times 10^{-3}\left({ }^{3}\right) .
\end{aligned}
$$

D'après la manière dont elle a été déduite, on remarque que cette valeur est énorme dans la mesure où elle est seulement trois fois plus petite que la valeur maximale permise par la nature du processus et que la valeur correspondante pour la désintégration $\alpha$ de l'état fondamental est, quant à elle, trente ordres de grandeur plus petite.

Cette réussite à reproduire la valeur expérimentale de $N_{\alpha} / N_{\mathrm{F}}$ réduit en même temps les doutes associés à la détermination de la largeur réduite $\alpha$, ou plus précisément du facteur de transmission des particules $\alpha$ (voir aussi réf. [14]).

8. L'influence des caractéristiques du système fissionable. - En nous situant dans la ligne de la même hypothèse, il nous reste à étudier la dépendance du spectre énergétique et du rapport $N_{\alpha} / N_{\mathrm{F}}$ par rapport aux paramètres du système fissionable.

La valeur la plus probable de l'énergie cinétique est déterminée seulement par la hauteur de la barrière et par la vitesse de déplacement des fragments immédiatement après le moment d'émission. Elle sera, par conséquent, complètement indépendante de l'énergie d'excitation et relativement constante en fonction du paramètre de fissibilité, l'accroissement de l'accélération de préscission compensant l'élévation de la barrière.

En ce qui concerne la dépendance de l'énergie cinétique vis-à-vis du rapport des masses, la fonction $\vec{E}_{\alpha}(R)$ ne pourrait être calculée que si l'on connaissait exactement la modification du mode de scission en fonction de l'asymétrie. Une idée en est donnée par la grandeur $\bar{E}_{\mathrm{F}}(R)$ mesurée. Le fait que, quel que soit le système étudié, le maximum de cette fonction corresponde au fragment double magique ${ }^{132} \mathrm{Sn}$, suggère qu'il s'agit essentiellement d'un effet de forme et que par conséquent la variation des élongations commande principalement ce comportement. Mais puisque une augmentation de seulement $20 \%$ du rapport des masses devrait être accompagnée d'une diminution de $D_{0}$ de $3 \mathrm{fm}$ (qui est toutefois inconfortablement grande pour l'expliquer) il faut dès lors supposer que la modification de $E_{\mathrm{F}}^{0}$ apporte aussi une contribution. La variation de $\bar{E}_{\alpha}$ avec $R$ a été calculée, figure 4 , pour diverses hypothèses

$\left.{ }^{3}\right)$ On a constaté que la rupture des paires dans la fission en fragments impairs se fait sur le compte de l'énergie cinétique de préscission [13] et donc du ralentissement du processus de scission. Par conséquent on s'attend que $N_{\alpha} / N_{\mathrm{F}}$ montre un effet pair-impair avec les valeurs plus grandes correspondant aux divisions impaires. 


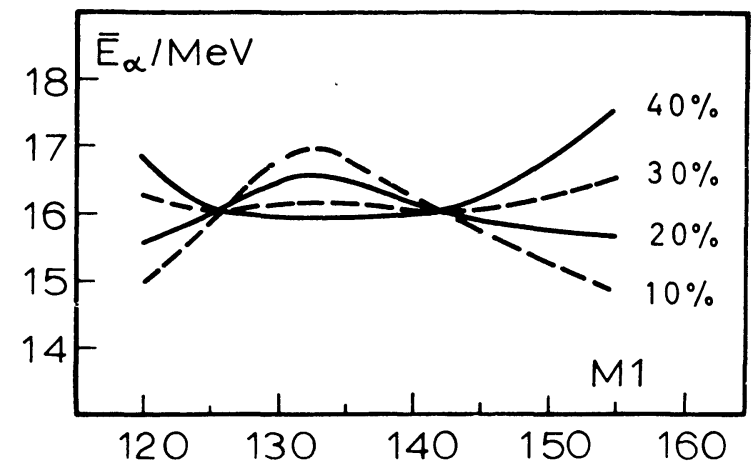

Fig. 4. - Dépendance de la valeur la plus probable de l'énergie cinétique des particules $\alpha$ émises pendant la scission de ${ }^{236} U$ en fonction de la masse du fragment lourd. On a considéré les cas dans lesquels $E_{\mathrm{F}}^{0}$ contribue pour $10,20,30$ et $40 \%$ à la variation de $E_{\mathrm{F}}$ en fonction de la masse du fragment lourd.

relatives à cette contribution. Il est sûrement très probable que la proportion $\Delta E_{\mathrm{F}}^{0} / \Delta E_{\mathrm{F}}$ est elle-même fonction de masse, en sorte que les courbes de la figure 4 ont seulement une valeur indicative $\left({ }^{4}\right)$.

Par contre, une mesure précise de cette dépendance peut être utilisée pour obtenir des informations quantitatives sur la modification du mode de scission en fonction de l'asymétrie.

La largeur du spectre énergétique des particules légères de scission subira les mêmes variations que la largeur de la distribution de l'énergie cinétique des fragments, c'est-à-dire qu'elle augmentera avec la température nucléaire et diminuera avec l'augmentation de l'asymétrie en accord avec ce que l'expérience indique [2].

En ce qui concerne les variations du rapport $N_{\alpha} / N_{\mathrm{F}}$ en fonction des divers paramètres, elles seront généralement petites et déterminées par les variations de la largeur réduite $\delta^{2}\left({ }^{5}\right)$.

De telles corrélations entre $\left(N_{\alpha} / N_{\mathrm{F}}\right)_{\text {exp. }}$ et $\delta^{2}$ ont été mises récemment en évidence [15].

La probabilité de formation d'amas diminuant en même temps que l'échauffement de la matière nucléaire, $N_{\alpha} / N_{\mathrm{F}}$ décroîtra légèrement avec l'énergie d'excitation.

On sait que $\delta^{2}$ varie peu d'un noyau à l'autre si ce n'est dans les rares cas où on franchit des intervalles séparant les couches énergétiques des protons ou des neutrons. Une étude systématique de la variation de $N_{\alpha} / N_{\mathrm{F}}$ avec $Z$ et $N$ pourrait donc confirmer ou infirmer l'existence de noyaux magiques aux formes à cou marqué [16], c'est-à-dire de couches fermées au-delà du dernier point-seuil.

Enfin, il faudrait peut-être ajouter que l'émission

$\left({ }^{4}\right)$ En dépit de ces incertitudes, le fait que se produise toujours un changement dans l'allure de la courbe à la valeur de $M=132$ demeure néanmoins une caractéristique générale.

$\left({ }^{5}\right)$ Cette affirmation ne s'applique qu'aux noyaux transuraniens pour lesquels la distribution de l'énergie disponible dépasse la dépense d'énergie $E_{\mathrm{c}}$. Pour les noyaux suburaniens, on doit s'attendre à ce que la proportion des particules $\alpha$ dans l'ensemble des fissions diminue pour devenir indétectable pour $Z^{2} / A^{1 / 3}<1000$. simultanée des deux particules légères peut aussi être traitée dans le même cadre, la probabilité de coexistence de deux particules dans un noyau étant finie.

$\bar{E}_{\alpha}$, mesuré dans le cas d'une telle émission double pendant la fission spontanée de ${ }^{252} \mathrm{Cf}$ [17], est de $2 \mathrm{MeV}$ plus petit que dans le cas de l'émission simple, ce qui reflète le fait que la déformation au moment de l'émission est plus grande et, peut-être, que $2 \times E_{c}^{\text {cou }}$ dépasse la moyenne de l'énergie disponible. Cette dernière supposition peut justifier aussi la valeur relativement petite de $N_{2 \alpha} / N_{\mathrm{F}}$ par rapport à $\left(N_{\alpha} / N_{\mathrm{F}}\right)^{2}$. En même temps, prédisant une proportion plus élevée des polaires dans l'ensemble des particules de scission, elle peut expliquer une valeur $W\left(180^{\circ}\right) / W\left(90^{\circ}\right)$ près de l'unité, c'est-à-dire la corrélation angulaire expérimentale [17], paradoxalement petite, entre les deux particules légères.

Pour la majorité des points étudiés l'accord entre les prévisions théoriques et les données expérimentales peut être vérifié consultant la réf. [4]. Les autres sources ont été citées séparément.

Quoique toutes les estimations précédentes soient en accord avec les données expérimentales existantes, on n'a pas voulu confronter ces calculs préliminaires directement avec les résultats expérimentaux. Pour assurer définitivement la validité du modèle présent, des calculs détaillés seront effectués.

9. Discussions. - Il est intéressant de remarquer que la différence entre les énergies d'excitation des fragments lourds dans les deux modes de fission (voir $\S 2$ ) ne dépasse pas visiblement la valeur $Q_{\alpha}^{0}$ au moment de la scission, au moins dans les limites entre lesquelles ces deux valeurs peuvent être estimées, et ne peut donc être interprétée comme l'énergie consommée à partir de l'énergie d'excitation pour l'émission de l'alpha. En d'autres termes, c'est l'énergie intrinsèque supplémentaire que le système fissionable, dans la mesure où il ne contient pas un amas $\alpha$ libre, peut transformer en énergie d'excitation.

L'énergie nécessaire $\left(E_{\mathrm{c}}\right)$ est donc dépensée sur le compte de l'énergie cinétique de préscission, ce qui est aussi en accord avec la valeur très petite $E_{\mathrm{F}}^{0}$, déduite dans le $\S 6$, en comparaison avec les $40 \mathrm{MeV}$ approximatifs déduits dans le cas de la fission binaire [10, 11]. Dans cette situation, l'énergie cinétique totale additionnelle mesurée dans la fission $\alpha$ représente justement $Q_{\alpha}^{0}$ au moment de l'émission :

$$
\left(\bar{E}_{\mathrm{F}}^{\alpha}-\bar{E}_{\mathrm{F}}\right)+\bar{E}_{\alpha}=Q_{\alpha}^{0} .
$$

Le même raisonnement est aussi valable pour l'émission polaire $\left(^{6}\right)$ et on a ainsi en principe trois points,

$\left.{ }^{6}\right)$ Dans ce cas, il n'existe pas encore de valeur expérimentale pour $\Delta \bar{E}_{\mathrm{F}}$. Par contre, on peut approcher $Q_{\alpha}^{0}$ au moment de l'émission polaire par sa valeur au moment de l'émission équatoriale $\left(2,5 \mathrm{MeV}\right.$ dans le cas de $\left.{ }^{236} \mathrm{U}\right)$ et estimer ainsi cette différence. Il en résulte une énergie cinétique totale des fragments dans le cas de l'émission d'un alpha polaire, inférieure de $20,5 \mathrm{MeV}$ à celle de la fission ordinaire. 
au-delà du dernier point-seuil, auxquels on peut tester l'applicabilité de nos méthodes de calcul des masses aux noyaux déformés au maximum.

La même remarque conduit aussi à la conclusion que la probabilité d'excitation interne pendant le processus de scission est très petite, au moins dans sa première partie. Dans le cas particulier des excitations des quasi-particules, ceci a déjà été démontré [13].

Pour terminer, il est souhaitable d'essayer de fournir une réponse à la question théorique essentielle de ce processus : pourquoi une si grande partie de l'énergie disponible est-elle cédée à la particule légère ?

Comme cela a déjà été remarqué les conditions suivantes doivent être satisfaites pour qu'il en soit ainsi :

a) L'énergie disponible doit être concentrée sur très peu de degrés de liberté.

De même que dans les autres modèles proposés, ce réservoir d'énergie initiale est formé par l'énergie de déformation. Mais ici c'est l'énergie de déformation du système fissionable au dernier point-seuil et non celle des fragments primaires, qui est considérée.

b) Le degré de liberté de la particule légère doit être fortement couplé avec ceux dans lesquels l'énergie disponible est concentrée.

Immédiatement après le point-seuil, comme dans tout processus relativement lent, les excitations intrinsèques sont peu probables, voir par exemple réf. $[18,19]$. Dans la mesure où une particule libre existe dans un noyau, le couplage mentionné plus haut est donc fort. En outre, le degré de liberté de l'alpha est également favorisé aux dépens du degré de liberté de la fission parce qu'il laisse aussi le mouvement du centre de masse dans son état fondamental. Le caractère approximativement adiabatique du pro- cessus de fission est donc conservé jusqu'au moment de la libération de la particule. Le processus se déroulera ensuite comme dans la fission ordinaire : si la vitesse dépasse la limite adiabatique, la probabilité d'excitation croît, ce qui ralentit à son tour l'accélération subséquente du processus.

Il existe, pour un certain aspect, une ressemblance frappante avec la désintégration $\alpha$ ordinaire d'un état initial vers différents états finaux : toute l'énergie libérée est généralement cédée à la particule $\alpha$ et seulement très rarement utilisée à l'excitation du noyau résiduel. Ceci découle de ce que les transitions qui impliquent des modifications essentielles dans l'état du noyau sont fortement interdites. On explique de même le minimum, dans la section efficace pour le transfert des deux particules, au point de transition sphérique-déformé [20].

$\mathrm{Si}$ elle est générale, cette explication pourrait contribuer à la formation d'une image plus concrète de ce qu'on nomme l'amortissement de la descente vers la scission : l'échange du degré d'excitation est influencé d'une part par le degré de non-adiabaticité de cette descente, d'autre part par la valeur maximale du recouvrement des états impliqués.

10. Conclusion. - On a montré que la compréhension du mode d'émission des particules légères de scission peut transformer ce processus en un véritable instrument d'étude de la structure et de la dynamique du système fissionable au-delà du dernier point-seuil.

Remerciements. - Je voudrais exprimer ma gratitude au Professeur J. Theobald pour l'intérêt qu'il a manifesté à ce travail et la lecture critique du manuscrit.

\section{Bibliographie}

[1] Schmitt, H. W., Neiler, J. H., Walter, F. J. et ChethamStrode, A., Phys. Rev. Lett. 9 (1962) 427.

[2] Metha, G. K., Poitou, J., Ribrag, M. et Signarbieux, C., Phys. Rev. C 7 (1973) 373.

[3] Piasecki, E. et Blocki, J., Nucl. Phys. A 208 (1973) 381.

[4] Halpern, I., Annu. Rev. Nucl. Sci. 21 (1971) 245.

[5] Wagemans, C. et Deruytter, A. J., Nucl. Phys. A 194 (1972) 657 et $A 212$ (1973) 556.

[6] Câruan, N., Săndulescu, A. et Pashkevich, V. V., Phys. Rev. C 11 (1975) 782.

[7] Nilsson, S. G., Tsang, C. F., Sobiczewski, A., Szymansky, Z., Wycech, S., Gustafson, C., Lamm, I. L., Möller, P. et Nilsson, B., Nucl. Phys. A 131 (1969) 1.

[8] Swiatecki, W. J. et BJornholm, S., Phys. Lett. 4C (1972) 325.

[9] Loveland, W., Phys. Rev. C 9 (1974) 395.

[10] Nix, J. R., Nucl. Phys. A 130 (1969) 241.

[11] Hasse, R. W., Nucl. Phys. A 128 (1969) 609.

[12] Rasmussen, J. O., Phys. Rev. 113 (1959) 1593.

[13] Nifenecker, H., Signarbieux, C., Babinet, R. et Poitou, J.,
Proceedings of the Third Symposium on Physics and Chemistry of Fission, Rochester, 1973 (IAEA, Vienna, 1974), Vol. II, p. 117.

[14] Câruan, N. et Săndulescu, A., Z. Naturforsch. 26a (1971) 1389.

[15] Wagemans, C. et Deruytter, A. J., Z. Phys. A 275 (1975) 149.

[16] Brack, M., Damgaard, J., Jensen, A. S., Pauli, H. C., Strutinsky, V. M. et Wong, C. Y., Rev. Mod. Phys. 44 (1972) 320.

[17] Kataria, S. K., Nardi, E. et Thompson, S. G., Proceedings of the Third Symposium on Physics and Chemistry of Fission, Rochester, 1973 (IAEA, Vienna, 1974), Vol. II, p. 389.

[18] Boneh, Y. et Fraenkel, Z., Phys. Rev. C 10 (1974) 893.

[19] Ledergerber, T., Paltiel, Z., Pauli, H. C., Schütte, G., Yariv, Y. et Fraenkel, Z., Phys. Lett. 56B (1975) 417.

[20] Kumar, K., The Electromagnetic Interaction in Nuclear Spectroscopy (North-Holland) 1975, p. 130. 